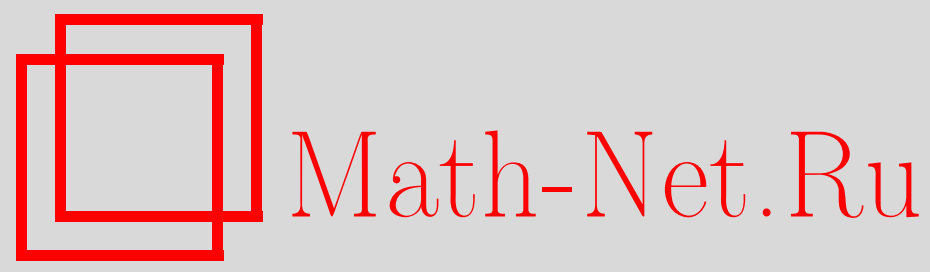

А. А. Злотник, Слабые решения уравнений движения вязкой сжимаемой реагирующей бинарной смеси: единственность и непрерывная по Липшицу зависимость от данных, Матем. заметки, 2004, том 75, выпуск 2, 307-310

DOI: https://doi.org/10.4213/mzm546

Использование Общероссийского математического портала Math-Net.Ru подразумевает, что вы прочитали и согласны с пользовательским соглашением http://www . mathnet.ru/rus/agreement

Параметры загрузки:

IP : 54.209 .52 .79

26 апреля 2023 г., 12:02:00

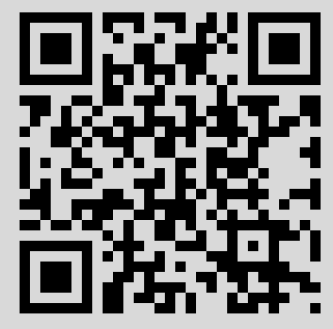




\section{СЛАБЫЕ РЕШЕНИЯ УРАВНЕНИЙ ДВИЖЕНИЯ ВЯЗКОЙ СЖИМАЕМОЙ РЕАГИРУЮЩЕЙ БИНАРНОЙ СМЕСИ: ЕДИНСТВЕННОСТЬ И НЕПРЕРЫВНАЯ ПО ЛИПШИЦУ ЗАВИСИМОСТЬ ОТ ДАННЫХ}

\section{А. А. Злотник}

1. Системам квазилинейньх уравнений движения вязких сжимаемых сред посвящена обширная литература [1], [2]. Одной из актуальных проблем является разработка теории глобальных по времени и по данньм слабых решений, включая вопросы их существования, единственности и непрерьвной зависимости от данных (начальных данных, коэффициентов уравнений, свободных членов и т.д.) и др. Для системы уравнений одномерного движения вязкого теплопроводного газа последние два вопроса изучались в [3]-[6] (см. также [7]) и [8]. Для более сложных систем уравнений для реагирующего газа (иными словами, в задаче горения) слабые решения рассмотрены в [9], [10].

Совсем недавно в [11] получено, в частности, существование слабых решений для еще более сложной системы уравнений для реагирующей бинарной газовой смеси; отметим, что регулярные решения задач о бинарных смесях ранее исследовались в [12], [13]. Настоящая работа посвящена вопросам единственности этих слабых решений и их непрерьвной зависимости от данных.

Система квазилинейных уравнений одномерного движения вязкой сжимаемой реагирующей бинарной газовой смеси имеет вид

$$
\begin{gathered}
\eta_{t}=u_{x}, \quad u_{t}=\sigma_{x}, \quad \sigma=\nu \rho u_{x}-p, \quad p=k(z) \rho \theta, \quad \rho=\eta^{-1}, \\
\left(c_{V}(z) \theta\right)_{t}=\left(\lambda \rho \theta_{x}\right)_{x}+\sigma u_{x}+\mu f(\theta), \quad z_{t}=-f(\theta) z
\end{gathered}
$$

в области $(x, t) \in Q=Q_{T} \equiv \Omega \times(0, T)$ при краевых и начальных условиях

$$
\left.u\right|_{x=0, M}=0,\left.\quad \lambda \rho \theta x\right|_{x=0, M}=0,\left.\quad(\eta, u, \theta, z)\right|_{t=0}=\left(\eta^{0}(x), u^{0}(x), \theta^{0}(x), z^{0}(x)\right) .
$$

Здесь $\Omega=(0, M)$, а $k(z)=k_{1} z+k_{2}(1-z), c_{V}(z)=c_{V 1} z+c_{V 2}(1-z)$, причем $\nu>0, \lambda>0$, $\mu>0, k_{i}>0, c_{V i}>0, i=1,2,-$ физические постоянные. Специфичным для задачи о бинарной смеси является зависимость коэффициентов $k$ и $c_{V}$ от функции $z$.

Искомые функции $\eta>0, u, \theta>0$ и $0 \leqslant z \leqslant 1$ - соответственно удельный объем, скорость, абсолютная температура и концентрация невыгоревшего топлива - являются функциями лагранжевых массовых координат $(x, t)$. Функции $\sigma, p, \rho$ - это соответственно напряжение, давление, плотность.

Нам потребуются пространства $L^{q, r}(Q), V_{2}(Q)[14]$ с нормами

$$
\|w\|_{L^{q, r}(Q)}=\|\| w\left\|_{L^{q}(\Omega)}\right\|_{L^{r}(0, T)}, \quad\|w\|_{V_{2}(Q)}=\|w\|_{L^{2, \infty}(Q)}+\left\|w_{x}\right\|_{L^{2}(Q)},
$$

где $q, r \in[1, \infty]$. Пусть также $W_{\mathbf{q}, \mathbf{r}}^{1,0}(Q)[4],[5]-$ пространство с нормой

$$
\|w\|_{W_{\mathbf{q}, \mathbf{r}}^{1,0}(Q)}=\|w\|_{L^{q_{0}, r_{0}(Q)}}+\left\|w_{x}\right\|_{L^{q_{1}, r_{1}(Q)}},
$$

где $\mathbf{q}=\left(q_{0}, q_{1}\right), \mathbf{r}=\left(r_{0}, r_{1}\right)$, a $H^{1}(\Omega)=W_{2}^{1}(\Omega)-$ пространство Соболева.

Предположим, что начальные данные обладают свойствами $\eta^{0}, z^{0} \in L^{\infty}(\Omega), u^{0}, \theta^{0} \in L^{2}(\Omega)$ и $\operatorname{ess}_{\Omega} \inf _{\Omega} \eta^{0}>0, \theta^{0}>0,0 \leqslant z^{0} \leqslant 1$, a $f \in C^{1}([0, \infty)), 0 \leqslant f(\theta) \leqslant \bar{f}$. Будем изучать слабое решение задачи (1)-(3) (кратко- задачи $\mathscr{P}$ ) со свойствами (несколько более широкими, чем в [11])

$$
\eta, z \in L^{\infty}(Q), \quad \eta_{t}, z_{t} \in L^{2}(Q), \quad u, \theta \in V_{2}(Q), \quad \operatorname{essinf} \eta>0, \quad \theta>0, \quad 0 \leqslant z \leqslant 1 .
$$

Работа выполнена при финансовой поддержке Российского фонда фундаментальных исследований, грант № 01-01-00700. 
Отметим, что свойства $\theta^{0}>0$ и $\theta>0$ ниже фактически несущественны.

Рассмотрим два экземпляра - $\mathscr{P}^{(1)}$ и $\mathscr{P}^{(2)}$ - задачи $\mathscr{P}$, в которых решения, функции $\sigma, p, \rho, f$, коэффициенты и начальные данные будем помечать верхним индексом (1) и (2). Введем операцию взятия разности $\Delta \varphi:=\varphi^{(1)}-\varphi^{(2)}$, так что $\Delta \eta=\eta^{(1)}-\eta^{(2)}, \Delta \eta^{0}=\eta^{0,(1)}-\eta^{0,(2)}, \Delta \nu=\nu^{(1)}-$ $\nu^{(2)}$ и т.д.

Пусть $\bar{N} \geqslant N>1$ - параметры. Через $K=K(\bar{N}), K_{1}=K_{1}(\bar{N}), \ldots$ будем обозначать положительные неубьвающие функции от параметра $\bar{N}$, которые могут зависеть также от $M, T$, но не от данных рассматриваемых задач. Положим также $\|y\|_{C}=\sup _{\zeta \geqslant 0}|y(\zeta)|$ и $\|y\|_{C^{1}}=\|y\|_{C}+$ $\left\|y^{\prime}\right\|_{C}$.

Теорема. Пусть при $\ell=1,2$ коэффичиенты и начальные данные удовлетворяют условиям

$$
\begin{gathered}
\nu^{(\ell)}, k_{i}^{(\ell)}, c_{V i}^{(\ell)}, \lambda^{(\ell)}, \mu^{(\ell)} \in\left[N^{-1}, N\right], \quad i=1,2, \quad\left\|f^{(\ell)}\right\|_{C^{1}} \leqslant N, \\
N^{-1} \leqslant \eta^{0,(\ell)} \leqslant N, \quad\left\|u^{0,(\ell)}\right\|_{L^{2}(\Omega)}+\left\|z^{0,(\ell)}\right\|_{H^{1}(\Omega)} \leqslant N .
\end{gathered}
$$

Пусть также $\bar{N}^{-1} \leqslant \eta^{0,(\ell)} \leqslant \bar{N},\left\|u^{(\ell)}\right\|_{V_{2}(Q)}+\left\|\theta^{(\ell)}\right\|_{V_{2}(Q)} \leqslant \bar{N}$. Тогда справедлива следующал оценка нормы разности слабых решений задач $\mathscr{P}^{(1)}$ и $\mathscr{P}^{(2)}$ :

$$
\begin{aligned}
& \|\Delta \eta\|_{L^{\infty}(Q)}+\|\Delta u\|_{V_{2}(Q)}+\|\Delta \theta\|_{W_{\mathbf{q}, \mathbf{r}}^{1,0}(Q)}+\|\Delta z\|_{L^{\infty}(Q)}+\left\|\Delta z_{x}\right\|_{L^{2, \infty}(Q)} \\
& \leqslant K_{\varepsilon}(N)\left(\Delta^{0}+\Delta_{c}+\|\Delta f\|_{C^{1}}\right)
\end{aligned}
$$

через нормы разностей начальных данных и коэффициентов

$$
\begin{aligned}
& \Delta^{0}:=\left\|\Delta \eta^{0}\right\|_{L^{\infty}(\Omega)}+\left\|\Delta u^{0}\right\|_{L^{2}(\Omega)}+\left\|\Delta \theta^{0}\right\|_{L^{1}(\Omega)}+\left\|\Delta z^{0}\right\|_{H^{1}(\Omega)}, \\
& \Delta_{c}:=|\Delta \nu|+\left|\Delta k_{1}\right|+\left|\Delta k_{2}\right|+\left|\Delta c_{V 1}\right|+\left|\Delta c_{V 2}\right|+|\Delta \lambda|+|\Delta \mu|,
\end{aligned}
$$

при всех $\mathbf{q}=\left(q_{0}, q_{1}\right), \mathbf{r}=\left(r_{0}, r_{1}\right) \operatorname{ma\kappa ux,~что~} q_{0}, r_{0} \in[1, \infty], q_{1}, r_{1} \in[1,2] u 1 /\left(2 q_{0}\right)+$ $1 / r_{0}=(1+\varepsilon) / 2\left(\right.$ или $\left.q_{0}=1, r_{0}=\infty\right), 1 /\left(2 q_{1}\right)+1 / r_{1}=1+\varepsilon / 4$ с любым $\varepsilon>0$.

СледствиЕ. Если $z^{0} \in H^{1}(\Omega)$, то слабое решение задачи (1)-(3) единственно.

2. Для доказательства теоремы применим подход из [5]. Для краткости будем считать, что $\mathscr{P}^{(1)}=\mathscr{P}$, т.е. верхний индекс (1) будем опускать. Положим $I_{0} y(t):=\int_{0}^{t} y(\tau) d \tau$. Ясно, что $z=e^{-I_{0} f(\theta)} z^{0}$, и так как $z^{0} \in H^{1}(\Omega)$, то существует

$$
z_{x}=e^{-I_{0} f(\theta)}\left[z_{x}^{0}-I_{0}\left(f^{\prime}(\theta) \theta_{x}\right) \cdot z^{0}\right]
$$

откуда $\left\|z_{x}\right\|_{L^{2, \infty}(Q)} \leqslant\left\|z_{x}^{0}\right\|_{L^{2}(\Omega)}+L\left\|\theta_{x}\right\|_{L^{2,1}(Q)}$, где $L=\left\|f^{\prime}\right\|_{C}$.

Установим сначала вспомогательные оценки для $\Delta \eta$ и $\Delta u$.

Лемма 1. При всех $0<t \leqslant T$ справедливы оченки

$$
\begin{gathered}
\|\Delta \eta\|_{L^{\infty}\left(Q_{t}\right)} \leqslant K\left(\|\Delta u\|_{L^{2, \infty}\left(Q_{t}\right)}+\|\Delta z\|_{L^{\infty, 2}\left(Q_{t}\right)}+\|\Delta \theta\|_{L^{\infty, 1}\left(Q_{t}\right)}\right. \\
\left.+\left\|\Delta \eta^{0}\right\|_{L^{\infty}(\Omega)}+\left\|\Delta u^{0}\right\|_{L^{2}(\Omega)}+\Delta_{1 c}\right), \\
\|\Delta u\|_{V_{2}\left(Q_{t}\right)} \leqslant K\left(\|d \Delta \eta\|_{L^{\infty, 2}\left(Q_{t}\right)}+\|\Delta z\|_{L^{2, \infty}\left(Q_{t}\right)}+\|\Delta \theta\|_{L^{2}\left(Q_{t}\right)}+\left\|\Delta u^{0}\right\|_{L^{2}(\Omega)}+\Delta_{1 c}\right),
\end{gathered}
$$

әде функиия $d \in L^{2}(0, T)$ такова, что $\|d\|_{L^{2}(0, T)} \leqslant K_{1}, u \Delta_{1 c}:=|\Delta \nu|+\left|\Delta k_{1}\right|+\left|\Delta k_{2}\right|$. Здесь и ниже величины $K, K_{1}$ не зависят от $t$. 
ДоКАЗАТЕльСТвО. Ясно, что $\Delta p=\left[k(z) \Delta \rho+\Delta k(z) \cdot \rho^{(2)}\right] \theta^{(2)}+k(z) \rho \Delta \theta$ и

$$
|\Delta k(z)| \leqslant\left|k_{1}-k_{2}\right| \cdot|\Delta z|+\left|\Delta k_{1}\right|+\left|\Delta k_{2}\right| \text {. }
$$

В силу равномерных двусторонних оценок $\eta^{(\ell)}$ и $z^{(\ell)}$ получим

$$
|\Delta p| \leqslant K\left[\theta^{(2)}|\Delta \eta|+\theta^{(2)}\left(|\Delta z|+\left|\Delta k_{1}\right|+\left|\Delta k_{2}\right|\right)+\Delta \theta\right] .
$$

Положив $d_{1}:=\left\|\theta^{(2)}\right\|_{L^{\infty}(\Omega)}$ и применив оценку $\left\|d_{1}\right\|_{L^{2}(0, T)}=\left\|\theta^{(2)}\right\|_{L^{\infty, 2}(Q)} \leqslant K$, вьведем

$$
\begin{aligned}
I_{0}\|\Delta p\|_{L^{\infty}(\Omega)} & \leqslant K\left[I_{0}\left(\left\|d_{1} \Delta \eta\right\|_{L^{\infty}(\Omega)}\right)+\|\Delta z\|_{L^{\infty, 2}\left(Q_{t}\right)}+\Delta_{1 c}+\|\Delta \theta\|_{L^{\infty, 1}\left(Q_{t}\right)}\right] \\
\|\Delta p\|_{L^{2}\left(Q_{t}\right)} & \leqslant K\left(\left\|d_{1} \Delta \eta\right\|_{L^{2}\left(Q_{t}\right)}+\|\Delta z\|_{L^{2, \infty}\left(Q_{t}\right)}+\Delta_{1 c}+\|\Delta \theta\|_{L^{2}\left(Q_{t}\right)}\right) .
\end{aligned}
$$

С учетом двух последних оценок доказательство леммы повторяет доказательства лемм 2 и 3 в [5].

Выведем теперь вспомогательные оценки для $\Delta z$.

ЛЕмма 2. При всех $0<t \leqslant T$ справедливы оценки

$$
\begin{aligned}
\|\Delta z\|_{L^{\infty}\left(Q_{t}\right)} & \leqslant K\left(\|\Delta \theta\|_{L^{\infty, 1}\left(Q_{t}\right)}+\left\|\Delta z^{0}\right\|_{L^{\infty}(\Omega)}+\|\Delta f\|_{C}\right), \\
\left\|\Delta z_{x}\right\|_{L^{2, \infty}\left(Q_{t}\right)} & \leqslant K\left(\|\Delta \theta\|_{L^{\infty, 1}\left(Q_{t}\right)}+\|\Delta \theta\|_{L^{2,1}\left(Q_{t}\right)}+\left\|\Delta z^{0}\right\|_{H^{1}(\Omega)}+\|\Delta f\|_{C^{1}}\right) .
\end{aligned}
$$

ДокАЗАТЕльСтво. Поскольку $z=e^{-I_{0} f(\theta)} z^{0}, f(\theta) \geqslant 0$ и $0 \leqslant z^{0,(2)} \leqslant 1$, то

$$
|\Delta z| \leqslant\left|\Delta I_{0} f(\theta)\right| z^{0,(2)}+e^{-I_{0} f(\theta)}\left|\Delta z^{0}\right| \leqslant L I_{0}|\Delta \theta|+T\|\Delta f\|_{C}+\left|\Delta z^{0}\right| .
$$

В силу формулы (5) имеем также

$$
\begin{aligned}
\left|\Delta z_{x}\right| \leqslant & \left|\Delta I_{0} f(\theta)\right|\left(\left|z_{x}^{0}\right|+\left|I_{0}\left(f^{\prime}(\theta) \theta_{x}\right)\right|\right)+e^{-I_{0} f^{(2)}\left(\theta^{(2)}\right)}\left[\left|\Delta z_{x}^{0}\right|\right. \\
& \left.+\left|I_{0} \Delta\left(f^{\prime}(\theta) \theta_{x}\right)\right|+\left|I_{0}\left(f^{\prime}(\theta) \theta_{x}\right)\right| \cdot\left|\Delta z^{0}\right|\right] \\
\leqslant & \left(L I_{0}|\Delta \theta|+T\|\Delta f\|_{C}\right)\left(\left|z_{x}^{0}\right|+L I_{0}\left|\theta_{x}\right|\right) \\
& +\left|\Delta z_{x}^{0}\right|+L I_{0}\left|\Delta \theta_{x}\right|+\left(T\left\|\Delta f^{\prime}\right\|_{C}+L\left|\Delta z^{0}\right|\right) I_{0}\left|\theta_{x}\right| .
\end{aligned}
$$

Полученные оценки для $\Delta z$ и $\Delta z_{x}$ влекут (8) и (9) соответственно (поскольку $\left\|z_{x}^{0}\right\|_{L^{2}(\Omega)}+$ $\left.\left\|\theta_{x}\right\|_{L^{2,1}(Q)} \leqslant K\right)$.

Дадим комбинированную оценку для $\Delta \eta, \Delta u$ и $\Delta z$.

ЛеммА 3. При всех $0<t \leqslant T$ справедлива оиенка

$$
\begin{gathered}
\|\Delta \eta\|_{L^{\infty}\left(Q_{t}\right)}+\|\Delta u\|_{V_{2}\left(Q_{t}\right)}+\|\Delta z\|_{L^{\infty}\left(Q_{t}\right)} \leqslant K\left(\|\Delta \theta\|_{L^{\infty, 1}\left(Q_{t}\right)}+\|\Delta \theta\|_{L^{2}\left(Q_{t}\right)}\right. \\
\left.+\left\|\Delta \eta^{0}\right\|_{L^{\infty}(\Omega)}+\left\|\Delta u^{0}\right\|_{L^{2}(\Omega)}+\left\|\Delta z^{0}\right\|_{L^{\infty}(\Omega)}+\Delta_{1 c}+\|\Delta f\|_{C}\right) .
\end{gathered}
$$

ДоКАЗАТЕЛЬСтво. Нормы $\Delta z$ в правых частях оценок леммы 1 можно оценить в силу (8). Тогда оценка (10) фактически следует из леммы 4 в [5].

Выведем ключевую оценку для $\Delta \theta$.

Лемма 4. При всех $0<t \leqslant T$ справедлива оченка

$$
\begin{aligned}
\|\Delta \theta\|_{W_{\mathbf{q}, \mathbf{r}\left(Q_{t}\right)}^{1,0}} \leqslant K & \left.K\|\Delta \eta\|_{L^{\infty}\left(Q_{t}\right)}+\|\Delta u\|_{V_{2}\left(Q_{t}\right)}+\|\Delta z\|_{L^{\infty}\left(Q_{t}\right)}+\left\|\Delta z_{x}\right\|_{L^{2, \infty}\left(Q_{t}\right)}\right) \\
& \left.+\|\Delta \theta\|_{L^{\infty, 1}\left(Q_{t}\right)}+\|\Delta \theta\|_{L^{2}\left(Q_{t}\right)}+\left\|\Delta \theta^{0}\right\|_{L^{1}(\Omega)}+\Delta_{c}+\|\Delta f\|_{C}\right),
\end{aligned}
$$

при всех $\mathbf{q}, \mathbf{r}$, указанных в теореме. 
ДоКАЗАТЕльство. Разделив первое из уравнений $(2)$ на $c_{V}(z)$ и использовав второе, формально получим $\left(\mathrm{c} c_{V}^{\prime}=c_{V 1}-c_{V 2}\right)$

$$
\theta_{t}=\left(\frac{\lambda \rho}{c_{V}(z)} \theta_{x}\right)_{x}+\frac{\lambda \rho c_{V}^{\prime}}{c_{V}^{2}(z)} z_{x} \theta_{x}+\frac{\sigma u_{x}}{c_{V}(z)}+\left(\mu+c_{V}^{\prime} \theta\right) f(\theta) \frac{z}{c_{V}(z)}
$$

Фактически $\theta \in V_{2}(Q)$ является слабым решением параболического уравнения (12) при краевых и начальных условиях

$$
\left.\frac{\lambda \rho}{c_{V}(z)} \theta_{x}\right|_{x=0, M}=0 \quad \text { и }\left.\quad \theta\right|_{t=0}=\theta^{0}
$$

что выводится при помощи стандартной работы с интегральными тождествами.

Поэтому $\Delta \theta \in V_{2}(Q)$ является слабым решением параболической задачи

$$
\begin{gathered}
\Delta \theta_{t}=\left(\beta \Delta \theta_{x}+\Psi\right)_{x}+F \quad \text { в } \quad Q, \\
\left.\left(\beta \Delta \theta_{x}+\Psi\right)\right|_{x=0, M}=0,\left.\quad \Delta \theta\right|_{t=0}=\Delta \theta^{0}
\end{gathered}
$$

с коэффициентом $\beta:=\lambda \rho / c_{V}(z)$ и свободными членами $\Psi:=\Delta \beta \cdot \theta^{(2)}$ и

$$
F:=F_{1}+F_{2}+F_{3}=\Delta\left(\frac{\lambda \rho c_{V}^{\prime}}{c_{V}^{2}(z)} z_{x} \theta x\right)+\Delta \frac{\sigma u_{x}}{c_{V}(z)}+\Delta\left(\left(\mu+c_{V}^{\prime} \theta\right) f(\theta) \frac{z}{c_{V}(z)}\right)
$$

Оценим нормы $\Psi$ и $F_{j}, j=1,2,3$. При всех $0<t \leqslant T$, использовав оценку $\left\|\theta_{x}^{(2)}\right\|_{L^{2}(Q)} \leqslant \bar{N}$, получим

$$
\|\Psi\|_{L^{2}\left(Q_{t}\right)} \leqslant \bar{N}\left\|\Delta \frac{\lambda \rho}{c_{V}(z)}\right\|_{L^{\infty}\left(Q_{t}\right)} \leqslant K\left(\|\Delta \eta\|_{L^{\infty}\left(Q_{t}\right)}+\|\Delta z\|_{L^{\infty}\left(Q_{t}\right)}+\Delta_{2 c}\right)
$$

с $\Delta_{2 c}:=|\Delta \lambda|+\left|\Delta c_{V 1}\right|+\left|\Delta c_{V 2}\right|$, cp. с (6). Поскольку

$$
F_{1}=\Delta \frac{\lambda \rho c_{V}^{\prime}}{c_{V}^{2}(z)} \cdot z_{x}^{(2)} \theta_{x}^{(2)}+\frac{\lambda \rho c_{V}^{\prime}}{c_{V}^{2}(z)}\left(\Delta z_{x} \cdot \theta_{x}+z_{x}^{(2)} \Delta \theta_{x}\right)
$$

а также $\left\|z_{x}^{(2)}\right\|_{L^{2, \infty}(Q)}+\left\|\theta_{x}\right\|_{L^{2}(Q)} \leqslant K$, вьведем

$$
\left\|F_{1}\right\|_{L^{1}\left(Q_{t}\right)} \leqslant K\left(\|\Delta \eta\|_{L^{\infty}\left(Q_{t}\right)}+\|\Delta z\|_{L^{\infty}\left(Q_{t}\right)}+\Delta_{2 c}+\left\|\Delta z_{x}\right\|_{L^{2, \infty}\left(Q_{t}\right)}+\left\|\Delta \theta_{x}\right\|_{L^{2,1}\left(Q_{t}\right)}\right)
$$

ср. с (15). В силу формулы

$$
F_{3}=\Delta\left(\left(\mu+c_{V}^{\prime} \theta\right) f(\theta)\right) \cdot \frac{z^{(2)}}{c_{V}^{(2)}\left(z^{(2)}\right)}+\left(\mu+c_{V}^{\prime} \theta\right) f(\theta) \Delta \frac{z}{c_{V}(z)}
$$

и оценки $\|\theta\|_{L^{2}(Q)} \leqslant K$ имеем

$$
\left\|F_{3}\right\|_{L^{2}\left(Q_{t}\right)} \leqslant K\left(\|\Delta \theta\|_{L^{2}\left(Q_{t}\right)}+\|\Delta z\|_{L^{\infty}\left(Q_{t}\right)}+|\Delta \mu|+\Delta_{2 c}+\|\Delta f\|_{C}\right) .
$$

Далее, приняв во внимание оценку $\left\|\Delta c_{V}\right\|_{L^{\infty}\left(Q_{t}\right)} \leqslant\left|c_{V 1}-c_{V 2}\right||| \Delta z \|_{L^{\infty}\left(Q_{t}\right)}+\left|\Delta c_{V 1}\right|+\left|\Delta c_{V 2}\right|$ и вторую оценку из (7), из доказательства леммы 6 в [5] (см. там оценки (25) и (26)) получим также

$$
\begin{aligned}
\left\|F_{2}\right\|_{L^{1}\left(Q_{t}\right)} \leqslant & K\left(\|\Delta \eta\|_{L^{\infty}\left(Q_{t}\right)}+\left\|\Delta u_{x}\right\|_{L^{2}\left(Q_{t}\right)}+\|\Delta \theta\|_{L^{2}\left(Q_{t}\right)}+\|\Delta z\|_{L^{\infty}\left(Q_{t}\right)}\right. \\
& \left.+\Delta_{1 c}+\left|\Delta c_{V 1}\right|+\left|\Delta c_{V 2}\right|\right) .
\end{aligned}
$$


Применив для линейной параболической задачи $(13),(14)$ стандартную $V_{2}\left(Q_{t}\right)$-оценку (в случае $\left.\boldsymbol{F}=0, \Delta \theta^{0}=0\right)$ и $W_{\mathbf{q}, \mathbf{r}}^{1,0}\left(Q_{t}\right)$-оценку из [4] (в случае $\left.\Psi=0\right)$, установим

$$
\|\Delta \theta\|_{W_{\mathbf{q}, \mathbf{r}\left(Q_{t}\right)}^{1,0}} \leqslant K\left(\|\Psi\|_{L^{2}\left(Q_{t}\right)}+\|F\|_{L^{1}\left(Q_{t}\right)}+\left\|\Delta \theta^{0}\right\|_{L^{1}(\Omega)}\right) .
$$

В силу указанных выше оценок для $\Psi$ и $F_{i}$ отсюда следует оценка (11).

В завершение доказательства теоремы подставим оценки (10) и (9) в правую часть (11) и получим при всех $0<t \leqslant T$ :

$$
\|\Delta \theta\|_{W_{\mathbf{q}, \mathbf{r}}^{1,0}\left(Q_{t}\right)} \leqslant K\left(\|\Delta \theta\|_{L^{2}\left(Q_{t}\right)}+\|\Delta \theta x\|_{L^{2,1}\left(Q_{t}\right)}+\Delta^{0}+\Delta_{c}+\|\Delta f\|_{C^{1}}\right) .
$$

Выбрав сначала $\mathbf{q}=(2,2)$ и $r_{0}>2, r_{1}>1$ и применив лемму 3.6 из [4] (которая заменяет в данной ситуации лемму Гронуолла), вьведем

$$
\|\Delta \theta\|_{L^{2}\left(Q_{t}\right)}+\|\Delta \theta\|_{L^{2,1}\left(Q_{t}\right)} \leqslant K\left(\Delta^{0}+\Delta_{c}+\|\Delta f\|_{C^{1}}\right) .
$$

Теперь оценки (10), (16) и (9) влекут оценку (4).

ЗАмЕч АниЕ. Как и в [5], можно рассмотреть неоднородные краевые условия (3), другие неоднородные краевые условия и учесть свободные члены во втором уравнении (1) и первом уравнении (2). Это не было сделано, чтобы не загромождать рассуждения.

\section{СПИСОК ЦИТИРОВАННОЙ ЛИТЕРАТУРЫ}

1. Антонцев С.Н., Кажихов А. В., Монахов В.Н. Краевые задачи механики неоднородных жидкостей. Новосибирск: Наука, 1983. 2. Lions P. L. Mathematical Topics in Fluid Mechanics. Vol. 2. Compressible Models. Oxford: Clarendon Press, 1998. 3. Амосов А. А., Злотник А. А. // Докл. АН СССР. 1988. Т. 301. №1. С. 11-15. 4. Злотник А. А., Амосов А. А. // Сиб. матем. ж. 1997. Т. 38. №4. С. 767-789. 5. Злотник А. А., Амосов А. А. // Матем. заметки. 1998. Т. 63. №6. С. 835-846. 6. Злотник А. А., Сун Цзян // Матем. заметки. 2003. T. 73. № 6. C. 795-800. 7. Zlotnik A. A., Amosov A. A. // The Navier-Stokes Equations: Theory and Numerical Methods / ed. R. Salvi. Lecture Notes in Pure and Appl. Math.. V. 223. New York-Basel: M. Dekker, 2001. P. 141-158. 8. Hoff D. // SIAM J. Math. Anal. 1996. V. 27. № 5. Р. 1193-1211. 9. Злотник А. А., Пузанов С. Н. // Матем. заметки. 1999. Т. 65. №6. С. $944-948$. 10. Chen G.-Q., Hoff D., Trivisa K. // Acta Math. Appl. Sinica. 2002. V. 18. № 1. P. 15-36. 11. Chen G.-Q., Hoff D., Trivisa K. // Arch. Rat. Mech. Anal. 2003. V. 166. № 4. P. 321-358. 12. Tabata M. // Kobe J. Math. 1992. V. 9. № 2. P. 195-205. 13. Петров А. Н. // Динамика сплошн. среды. 1993. №107. С. 112-123. 14. Ладыженская О.А., Солонников В.А., Уральцева Н. Н. Линейные и квазилинейные уравнения параболического типа. М.: Наука, 1968. 